\title{
Variáveis sociais, clínicas e hemodinâmicas associadas com sepse em pacientes na
}

\section{Unidade de Terapia Intensiva}

\author{
Social, clinical and hemodynamic variables associated with sepsis in patients at Intensive Care Unit \\ Variables sociales, clínicas y hemodinámicas asociadas a la sepsis en pacientes en la Unidad de \\ Cuidados Intensivos
}

Recebido: 05/01/2022 | Revisado: 10/01/2022 | Aceito: 18/01/2022 | Publicado: 19/01/2022

Edila Monteiro de Andrade

ORCID: https://orcid.org/0000-0002-5044-2291 Universidade Federal do Pará, Brasil E-mail: edilaandradee@gmail.com

Thalia Saraiva Mendonça

ORCID: https://orcid.org/0000-0002-7096-6367 Universidade Federal do Pará, Brasil

E-mail: thaliasaraiva03@gmail.com

Cleuma Oliveira Soares

ORCID: https://orcid.org/0000-0001-9869-620X Universidade Federal do Pará, Brasil E-mail: cleumaoliveira24@gmail.com

Giovana Salomão Melo

ORCID: https://orcid.org/0000-0003-0364-8526 Universidade Federal do Pará, Brasil E-mail: giovana.salomao@gmial.com

Isabella Boechat Faria Santos ORCID: https://orcid.org/0000-0001-8312-8752 Universidade Federal do Pará, Brasil E-mail: isabella.boechatfs@gmail.com João Simão de Melo-Neto ORCID: https://orcid.org/0000-0002-4681-8532 Universidade Federal do Pará, Brasil E-mail: jsmeloneto@ufpa.br

\begin{abstract}
Resumo
Objetivo: Analisar as variáveis sociais, clínicas e hemodinâmicas dos pacientes internados em Unidade de Terapia Intensiva (UTI) que evoluíram com sepse. Metodologia: Estudo retrospectivo, observacional e transversal. Foram elegíveis 224 prontuários, divididos em dois grupos: sepse presente (SP) $n=165$ e sepse ausente (SA) n=59. Foram descritas e analisadas as variáveis sociais, clínicas e hemodinâmicas. Resultados: As variáveis que tiveram associação com a sepse foram a baixa escolaridade e o estado civil casado. Nas variáveis clínicas observou-se que pacientes que precisam de intervenções invasivas foram associadas à sepse. As variáveis hemodinâmicas $\mathrm{PaCO} 2, \mathrm{Be}, \mathrm{Bicarbonato}$, leucócitos, hemácias, uréia, sódio e potássio alterados foram associados ao quadro de sepse. Estes pacientes permaneceram maior tempo na UTI e tiveram o óbito como desfecho. Conclusão: Existem variáveis sociais, clínicas e hemodinâmicas específicas que estão relacionadas à sepse. O conhecimento destas variáveis pode direcionar para que investimentos e recursos sejam direcionados visando prevenir esta complicação clínica.
\end{abstract}

Palavras-chave: Sepse; Síndrome de Resposta Inflamatória Sistêmica; Cuidados críticos.

\begin{abstract}
Objective: Analyze the social, clinical and hemodynamic variables of patients admitted to the Intensive Care Unit (ICU) who developed sepsis. Methodology: Retrospective, observational and cross-sectional study. 224 medical records were eligible, divided into two groups: present sepsis (SP) $n=165$ and absent sepsis (SA) $n=59$. Social, clinical and hemodynamic variables were described and analyzed. Results: The social variables low schooling and married marital status were associated with sepsis. In the clinical variables, it was observed that patients who need invasive interventions were associated with sepsis. The hemodynamic variables $\mathrm{PaCO} 2, \mathrm{Be}$, Bicarbonate, leukocytes, red blood cells, urea, sodium and potassium altered were associated with sepsis. These patients remained longer in the ICU and died more frequently. Conclusion: There are specific social, clinical and hemodynamic variables that are related to the presence of sepsis. Knowledge of these variables can guide investments and resources to be directed towards preventing this clinical complication.
\end{abstract}

Keywords: Sepsis; Systemic Inflammatory Response Syndrome; Critical care. 


\begin{abstract}
Resumen
Objetivo: Analizar las variables sociales, clínicas y hemodinámicas de los pacientes ingresados en la Unidad de Cuidados Intensivos (UCI) que desarrollaron sepsis. Metodología: Estudio retrospectivo, observacional y transversal. Fueron elegibles 224 registros médicos, divididos en dos grupos: sepsis presente (SP) n=165 y sepsis ausente (SA) n=59. Se describieron y analizaron variables sociales, clínicas y hemodinámicas. Resultados: Las variables que tenían una asociación con la sepsis eran la baja educación y el estado civil casado. En las variables clínicas, se observó que los pacientes que requirieron intervenciones invasivas se asociaron a sepsis. Las variables hemodinámicas $\mathrm{PaCO} 2, \mathrm{Be}$, Bicarbonato, leucocitos, glóbulos rojos, urea, sodio y potasio alterados se asociaron con sepsis. Estos pacientes permanecieron más tiempo en la UCI y tuvieron la muerte como resultado. Conclusión: Existen variables sociales, clínicas y hemodinámicas específicas que se relacionan con la sepsis. El conocimiento de estas variables puede orientar las inversiones y los recursos a orientar hacia la prevención de esta complicación clínica.
\end{abstract}

Palabras clave: Sepsis; Síndrome de Respuesta Inflamatoria Sistémica; Septicemia.

\title{
1. Introdução
}

Nas Unidades de Terapia Intensiva (UTI), a sepse é uma das principais causas de óbito após a internação, sendo altamente prevalente nos pacientes admitidos neste serviço (Genga \& Russell, 2017). Mesmo com avanços no serviço hospitalar, ainda é difícil reduzir os números de óbitos (Salomão, et al., 2019). Entre os motivos da alta mortalidade, sobreviventes de sepse frequentemente apresentam complicações clínicas, como infecções secundárias e alterações do sistema imunológico, levando a um quadro de reinternação (Genga \& Russell, 2017).

A sepse é responsável por mais de 10 milhões de mortes por ano e afeta de 3 a 10 pessoas nos países de alta renda, a cada 1.000 pacientes (Genga \& Russell, 2017). Ainda nos países desenvolvidos, como o Reino Unido, as taxas de mortalidade são aproximadamente 44.000 por ano (Szakmany et al., 2016). Em contrapartida, um levantamento nacional realizado pelo estudo Sepsis Prevalence Assessment Database (SPREAD) demonstrou incidência de 36,3 casos de sepse por 1.000 pacientes/dia em UTI no Brasil. Além disso, a taxa de mortalidade no Brasil por sepse é cerca de 55,7\%, com estimativa anual de mais de 200.000 mortes de pacientes adultos em tratamento de sepse (Machado, et al., 2017). Esses dados demonstram que há uma diferença no número de óbitos por sepse entre os países com diferentes níveis de desenvolvimento (Genga \& Russell, 2017).

A sepse é definida como o agravamento de um processo infeccioso acompanhado de uma resposta desregulada do sistema imunológico, levando à disfunção orgânica com risco de morte. É considerada uma síndrome influenciada por fatores patogênicos, como a virulência (Salomão, et al., 2019) e por características sociais e clínicas individuais dos pacientes, tais como: sexo, idade, origens genéticas e doenças subjacentes (Singer, et al., 2016; Marshall, 2018).

Pacientes com suspeita de infecção submetidos a cuidados intensivos e com aumento do tempo de internação estão mais vulneráveis ao desenvolvimento da sepse. Quando adquirida no ambiente hospitalar, a sepse está associada a maior taxa de mortalidade e aumento no tempo de internação (Westphal et al., 2019). Além dessa relação, outras variáveis clínicas também possuem associação com o desenvolvimento de sepse em UTI's, como o diagnóstico principal de doenças crônicas e comorbidades prévias (Dugani, et al., 2017; Tsertsvadze, et al., 2016). Tendo em vista os achados na literatura, notou-se que as características clínicas podem contribuir para a identificação da sepse, surgindo a necessidade de se conhecer melhor estas variáveis.

Outros fatores importantes são as características sociais, as quais estão relacionadas com diversos desfechos de saúde, como o envelhecimento saudável e o desenvolvimento de comorbidades, sendo possível analisar padrões associados (Engchuan, et al., 2019). Estudos evidenciam que variáveis sociais, como gênero, raça, idade e escolaridade estão relacionadas ao risco de adquirir sepse (Tsertsvadze, et al., 2016). Portanto, identificar as variáveis que podem influenciar no prognóstico de pacientes com sepse é essencial para a eficácia da prevenção, diagnóstico, tratamento e, consequentemente, redução das taxas de mortalidade (Da Silva, et al., 2019).

A análise hemodinâmica detecta se existe comprometimento da perfusão e da oxigenação dos tecidos e permite uma 
intervenção precoce a fim de prevenir ou reduzir a disfunção de órgãos e sistemas (consequência comum da evolução sepse) (De Figueiredo, et al., 2008). O estudo dessas variáveis pode contribuir com o diagnóstico das alterações hemodinâmicas e assim melhorar as medidas terapêuticas e o resultado de pacientes críticos (Antal, et al., 2020). Além da interpretação correta dos valores numéricos, o conhecimento dos mecanismos fisiopatológicos auxilia na avaliação das alterações hemodinâmicas dos pacientes com sepse grave (De Figueiredo, et al., 2008).

Diante dessas circunstâncias, este estudo teve por objetivo analisar as variáveis sociais, clínicas e hemodinâmicas na admissão e no desfecho dos pacientes que evoluíram com sepse durante internação em Unidade de Terapia Intensiva.

\section{Metodologia}

Estudo observacional e transversal (Schulz, et al., 2010). O processo de amostragem foi não probabilístico intencional, com a análise de prontuários de pacientes admitidos na Unidade de Terapia Intensiva do Hospital João de Barros Barreto, centro de referência terciária na região Norte do Brasil (Missão, 2019). Este estudo foi aprovado pelo Comitê de Ética em Pesquisa (CEP) (CAAE 82542017.7.0000.0017), respeitando os princípios éticos previstos na Resolução 466/12 do Conselho Nacional de Saúde / Conselho Nacional de Ética em Pesquisa.

A coleta de dados ocorreu no período de 2012 a 2018 e o recolhimento das informações pelos pesquisadores foi realizado no período de novembro de 2018 a maio de 2019, resultou em 224 prontuários elegíveis para o estudo, sendo 165 de pacientes com sepse, 59 pacientes que não desenvolveram sepse. Foram excluídos 4 prontuários por não apresentarem informações a respeito do desenvolvimento de sepse ou não durante a internação na UTI. Foram incluídos prontuários de pacientes admitidos na UTI e excluídos os que não passaram por este serviço, os que apresentavam dados insuficientes ou incompletos e os prontuários que não apresentavam informação sobre a sepse estar presente ou ausente. Os pacientes incluídos foram distribuídos nos grupos com sepse ausente (SA) e presente (SP).

Foram coletados os seguintes dados sociais desta população: idade, sexo, etnia, nível de escolaridade, profissão, religião e estado civil. As variáveis clínicas coletadas na admissão foram diagnóstico principal, nível de mobilidade, estado de gravidade admissional e nível de consciência. Também fizeram parte das variáveis clínicas o uso de oxigenoterapia, ventilação mecânica, frequência de aspiração brônquica, complicações clínicas durante a internação na UTI, uso de sondas, presença de edema, tempo de permanência na UTI e desfecho.

As variáveis hemodinâmicas foram coletadas na admissão (inicial) e desfecho (final), foram elas: creatina, potencial de hidrogênio $(\mathrm{pH})$, pressão parcial de gás carbônico $\left(\mathrm{PaCO}_{2}\right)$, bicarbonato $\left(\mathrm{HCO}_{3}\right)$, base $(\mathrm{Be})$, leucócitos, plaquetas, hemácias, ureia, sódio, potássio e lactato. Para os pacientes que apresentaram as variáveis hemodinâmicas na admissão e desfecho, foi analisada a variação $(\Delta)$, por meio da diferença entre os dados de desfecho e admissão ( $\Delta$ desfecho - $\Delta$ admissão).

Os dados foram submetidos à análise estatística descritiva e inferencial, descritos por meio de frequência absoluta $\mathrm{e}$ relativa (\%), média e desvio padrão (dados paramétricos), mediana e intervalo de confiança de 95\% (IC95\%) (dados nãoparamétricos). Para verificar a normalidade dos dados, as variáveis foram submetidas ao teste de Kolmogorov Smirnov. O nível de associação entre as variáveis categóricas foi analisado por meio do teste de qui-quadrado $\left(\chi^{2}\right)$, com correção de Yate, sendo o nível da associação determinado por Odds Ratio (OR), com IC 95\%. As variáveis numéricas foram analisadas por meio do teste t não-pareado (dados paramétricos) e de Mann-Whitney (dados não-paramétricos). Foi considerado estatisticamente significativo valores de $\mathrm{p} \leq 0,05$.

O poder do teste de $\chi^{2}(0,953)$ utilizado na maioria das análises inferenciais foi calculado com a variável mobilidade em pacientes acamados, selecionada de forma randomizada. Para o cálculo do poder do teste foi utilizado o valor de odds ratio $(3,444)$, proporção p2 $(0,678)$ e erro $\alpha$ de 0,05 . O software G*Power (3.1.9.2) foi utilizado para o cálculo. 


\section{Resultados}

Foram incluídos no estudo 224 prontuários, sendo 165 do grupo com sepse presente (SP) e 59 do grupo com sepse ausente (SA). No SP foi predominante o sexo feminino (51\%), enquanto no SA o gênero masculino foi o mais frequente (56\%). A média de idade não difere entre os grupos (SP: 50,35 $\pm 22,54$; SA: 46,49 $\pm 22,56 ; p=0,272$, teste $t$ não-pareado). $O$ tempo de permanência na UTI foi maior nos pacientes com sepse [SP: 8,0 (IC95\%: 10,770 - 15,191); SA: 5,0 (IC 95\%: 6,182 - 10,795); p = 0,035, teste de Mann-Whitney]. Logo, pacientes com sepse foram 18 vezes mais associados (IC95\%: 6,911 - 47,782) com o desfecho do óbito.

O resultado clínico, social e hemodinâmico do perfil dos pacientes sépticos que evoluíram à óbito e não sépticos que tiveram alta estão representados na Figura 1.

Figura 1. Perfil dos pacientes sépticos que evoluíram à óbito e não sépticos que tiveram alta hospitalar.
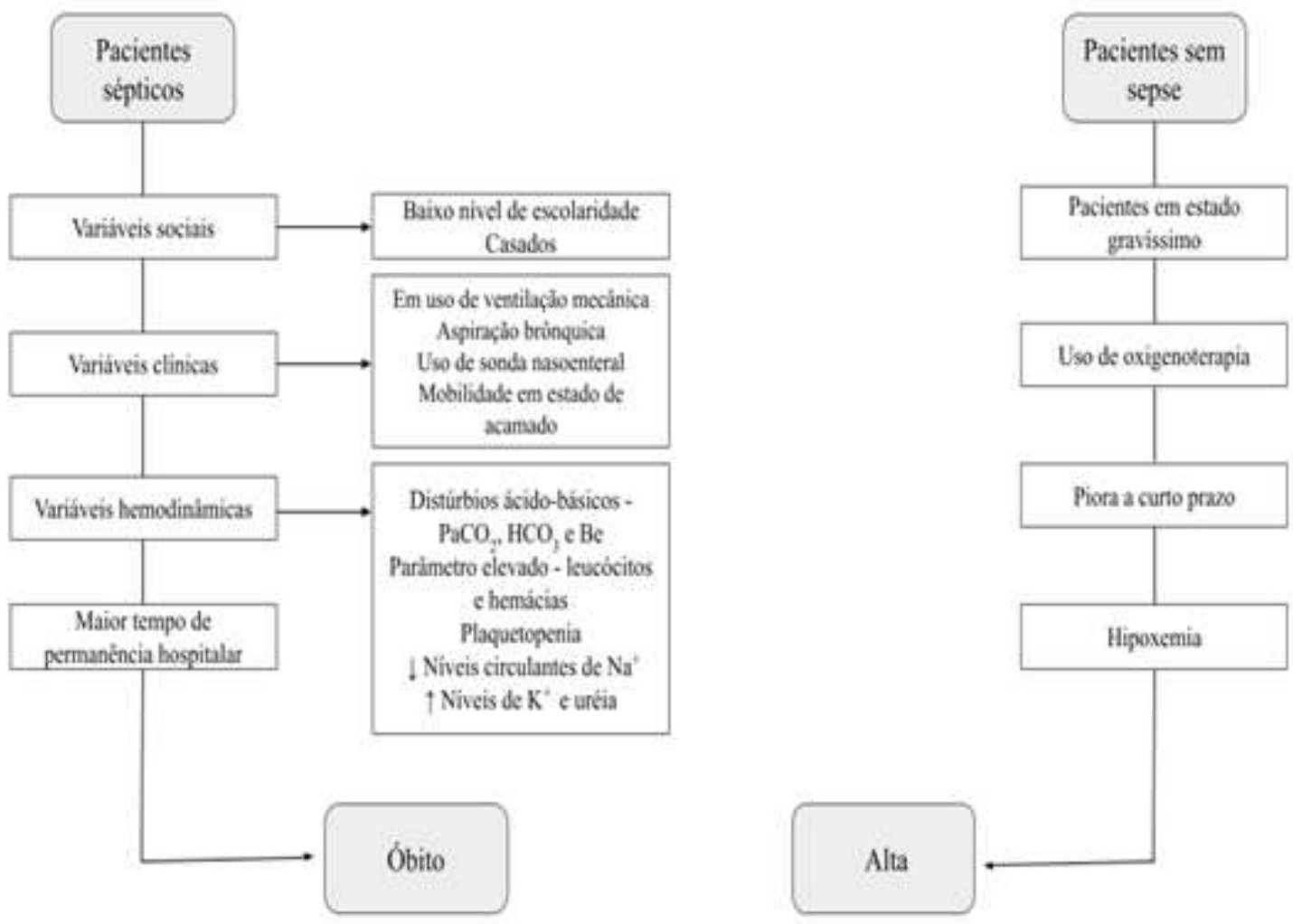

Fonte: Autores (2022).

As análises das variáveis sociais (Tabela 1), clínicas (Tabela 2) e hemodinâmicas (Tabela 3) entre os grupos, estão demonstradas nas respectivas tabelas. 
Tabela 1. Variáveis Sociais dos pacientes.

\begin{tabular}{|c|c|c|c|c|c|c|}
\hline Variáveis & Categorias & $\begin{array}{c}\text { SP } \\
(n=165)\end{array}$ & $\begin{array}{c}\text { SA } \\
(n=59)\end{array}$ & OR & IC95\% & $p$ \\
\hline \multirow[t]{2}{*}{ Sexo } & Feminino & $84(50,9)$ & $26(44,1)$ & & & \\
\hline & Masculino & $81(49,1)$ & $33(55,9)$ & 0,759 & $0,418-1,381$ & 0,453 \\
\hline \multirow[t]{5}{*}{ Etnia } & Branco & $27(16,4)$ & $9(15,3)$ & 1,087 & $0,478-2,470$ & 0,842 \\
\hline & Indígena & $0(0)$ & $1(1,7)$ & 0,117 & $0,005-2,935$ & 0,590 \\
\hline & Pardo & $121(73,3)$ & $37(62,7)$ & 1,635 & $0,870-3,072$ & 0,170 \\
\hline & Preto & $5(3)$ & $5(8,5)$ & 0,337 & $0,094-1,211$ & 0,170 \\
\hline & Não informado & $12(7,3)$ & $7(11,9)$ & & & \\
\hline \multirow[t]{7}{*}{ Nível de escolaridade } & Sem escolaridade & $115(9,1)$ & $2(3,4)$ & 5,800 & $0,749-44,933$ & 0,109 \\
\hline & $<09$ anos & $36(21,8)$ & $4(6,8)$ & 3,837 & $1,303-11,304$ & $0,016^{*}$ \\
\hline & 09 anos & $39(23,6)$ & $7(11,9)$ & 2,299 & $0,966-5,473$ & 0,083 \\
\hline & 10 a 11 anos & $0(0)$ & $0(0)$ & & & \\
\hline & 12 anos & $21(12,7)$ & $5(8,5)$ & 1,575 & $0,565-4,387$ & 0,523 \\
\hline & $>12$ anos & $12(7,3)$ & $1(1,7)$ & 4,549 & $0,578-35,791$ & 0,211 \\
\hline & Não informado & $42(25,5)$ & $40(67,8)$ & & & \\
\hline \multirow[t]{9}{*}{ Profissão } & Agricultor & $5(3)$ & $3(5,1)$ & 0,583 & $0,135-2,521$ & 0,748 \\
\hline & Aposentado & $8(4,8)$ & $2(3,4)$ & 1,452 & $0,299-7,045$ & 0,921 \\
\hline & Autônomo & $6(3,6)$ & $1(1,7)$ & 2,189 & $0,258-18,580$ & 0,764 \\
\hline & Do lar & $12(7,3)$ & $2(3,4)$ & 2,235 & $0,485-10,301$ & 0,456 \\
\hline & $\begin{array}{l}\text { Empregada } \\
\text { doméstica }\end{array}$ & $18(10,9)$ & $4(6,8)$ & 1,684 & $0,545-5,197$ & 0,509 \\
\hline & Estudante & $9(5,5)$ & $2(3,4)$ & 1,644 & $0,345-7,843$ & 0,780 \\
\hline & Lavrador & $8(4,8)$ & $0(0)$ & 6,422 & $0,365-113,09$ & 0,189 \\
\hline & Outros & $59(35,8)$ & $15(25,4)$ & & & \\
\hline & Não informado & $40(24,2)$ & $30(50,8)$ & & & \\
\hline \multirow[t]{7}{*}{ Religião } & Adventista & $2(1,2)$ & $0(0)$ & 1,820 & $0,086-38,482$ & 0,965 \\
\hline & Budista & $0(0)$ & $1(1,7)$ & 0,117 & $0,005-2,935$ & 0,590 \\
\hline & Católico & $12(7,3)$ & $2(3,4)$ & 2,235 & $0,485-10,301$ & 0,456 \\
\hline & Evangélico & $6(3,6)$ & $4(6,8)$ & 0,518 & $0,141-1,908$ & 0,524 \\
\hline & Metodista & $0(0)$ & $1(1,7)$ & 0,117 & $0,005-2,935$ & 0,590 \\
\hline & $\begin{array}{l}\text { Testemunha de } \\
\text { Jeová }\end{array}$ & $0(0)$ & $1(1,7)$ & 0,117 & $0,005-2,935$ & 0,590 \\
\hline & Umbanda & $1(0,6)$ & $0(0)$ & 1,085 & $0,044-27,026$ & 0,549 \\
\hline
\end{tabular}




\begin{tabular}{llllllll}
\hline Não informada & $143(86,7)$ & $50(84,7)$ & & & & & \\
Estado civil & Casado & $19(11,5)$ & $1(1,7)$ & 7,548 & $0,987-57,716$ & $0,045^{*}$ \\
Divorciado & $2(1,2)$ & $0(0)$ & 1,820 & $0,086-38,482$ & 0,965 \\
Solteiro & $47(28,5)$ & $14(23,7)$ & 1,280 & $0,643-2,549$ & 0,593 & 0,847 \\
& União estável & $38(23)$ & $15(25,4)$ & 0,877 & $0,441-1,748$ & 0,412 \\
Viúvo & $16(9,7)$ & $3(5,1)$ & 2,004 & $0,562-7,146$ &
\end{tabular}

SP: Sepse Presente. SA: Sepse Ausente. OR: Odds ratio. IC95\%: Intervalo de Confiança 95\%. *p<0,05, por meio do teste de qui-quadrado. Fonte: Autores (2022).

Tabela 2. Variáveis Clínicas dos pacientes.

\begin{tabular}{|c|c|c|c|c|c|c|c|}
\hline Variáveis & Categorias & & $\begin{array}{c}\text { SP } \\
(n=165)\end{array}$ & $\begin{array}{c}\text { SA } \\
(\mathbf{n}=59)\end{array}$ & OR & IC95\% & $p$ \\
\hline \multirow[t]{6}{*}{ Diagnóstico principal } & Câncer & & $38(23)$ & $15(25,4)$ & 0,878 & $0,441-1,748$ & 0,847 \\
\hline & $\begin{array}{l}\text { Doenças } \\
\text { respiratórias }\end{array}$ & & $38(23)$ & $8(13,6)$ & 1,907 & $0,833-4,370$ & 0,174 \\
\hline & SIDA & & $25(15,2)$ & $7(11,9)$ & 1,327 & $0,541-3,252$ & 0,687 \\
\hline & $\begin{array}{l}\text { Doenças } \\
\text { neurológicas }\end{array}$ & & $13(7,9)$ & $8(13,6)$ & 0,545 & $0,214-1,391$ & 0,305 \\
\hline & Pós-operatório & & $13(7,9)$ & $1(1,7)$ & 4,961 & $0,634-38,797$ & 0,170 \\
\hline & Outros & & $38(23)$ & $20(33,9)$ & & & \\
\hline \multirow[t]{3}{*}{ Mobilidade } & Acamado & & $145(87,9)$ & $40(67,8)$ & 3,444 & $1,678-7,068$ & $0,001 *$ \\
\hline & Deambulante & & $7(4,2)$ & $3(5,1)$ & 0,827 & $0,207-3,310$ & 0,788 \\
\hline & Não informado & & $13(7,9)$ & $16(27,1)$ & & & \\
\hline \multirow[t]{5}{*}{ Estado de gravidade } & Comprometido & & $2(1,2)$ & $0(0)$ & 1,820 & $0,086-38,482$ & 0,965 \\
\hline & Grave & & $12(7,3)$ & $5(8,5)$ & 0,847 & $0,285-2,516$ & 0,989 \\
\hline & Gravíssimo & & $20(12,1)$ & $16(27,1)$ & 0,370 & $0,177-0,777$ & $0,012 *$ \\
\hline & Regular & & $114(69,1)$ & $35(59,3)$ & 1,533 & $0,828-2,837$ & 0,228 \\
\hline & Não informado & & $7(4,2)$ & $3(5,1)$ & & & \\
\hline \multirow[t]{5}{*}{ Nível de consciência } & $\begin{array}{l}\text { Consciente } \\
\text { orientado }\end{array}$ & $\mathrm{e}$ & $72(43,6)$ & $23(39)$ & 1,043 & $0,565-1,926$ & 0,892 \\
\hline & $\begin{array}{l}\text { Consciente } \\
\text { desorientado }\end{array}$ & $\mathrm{e}$ & $8(4,8)$ & $7(11,9)$ & 0,366 & $0,126-1,061$ & 0,108 \\
\hline & Agitado & & $2(1,2)$ & $0(0)$ & 1,820 & $0,086-38,482$ & 0,965 \\
\hline & Torporoso & & $6(3,6)$ & $4(6,8)$ & 0,518 & $0,141-1,908$ & 0,524 \\
\hline & Coma não-induz & & $2(1,2)$ & $1(1,7)$ & 0,711 & $0,063-8,001$ & 0,781 \\
\hline
\end{tabular}




\begin{tabular}{|c|c|c|c|c|c|c|}
\hline & Coma induzido & $71(43)$ & $21(35,6)$ & 1,367 & $0,738-2,530$ & 0,399 \\
\hline & Não informado & $4(2,4)$ & $3(5,1)$ & & & \\
\hline Oxigenioterapia & & $10(6,1)$ & $21(35,6)$ & 0,117 & $0,051-0,268$ & $0,0001 *$ \\
\hline Ventilação Mecânica & & $152(92,1)$ & $43(72,9)$ & 4,351 & $1,942-9,746$ & $0,0004^{*}$ \\
\hline \multirow[t]{3}{*}{ Sondas } & Nasogástrica & $112(67,9)$ & $35(59,3)$ & 1,449 & $0,784-2,677$ & 0,304 \\
\hline & Vesical & $138(83,6)$ & $43(72,9)$ & 1,902 & $0,938-3,857$ & 0,107 \\
\hline & Nasoentérica & $65(39,4)$ & $6(10,2)$ & 5,742 & $2,334-14,127$ & $0,0001 *$ \\
\hline Edema presente & & $97(58,8)$ & $43(72,9)$ & 0,531 & $0,276-1,019$ & 0,078 \\
\hline Aspiração brônquica & & $121(73,3)$ & $28(47,5)$ & 3,045 & $1,643-5,641$ & $0,0006^{*}$ \\
\hline Complicações clínicas & & $71(43)$ & $18(30,5)$ & 1,720 & $0,912-3,244$ & 0,125 \\
\hline \multirow[t]{4}{*}{ Desfecho } & Óbito & $159(96,4)$ & $35(59,3)$ & 18,171 & $6,911-47,782$ & $0,0001 *$ \\
\hline & Alta & $3(1,8)$ & $10(16,9)$ & 0,090 & $0,024-0,343$ & $0,0001 *$ \\
\hline & Transferência & $3(1,8)$ & $1(1,7)$ & 1,074 & $0,109-10,538$ & 0,951 \\
\hline & Não Informados & $0(0)$ & $13(22)$ & & & \\
\hline
\end{tabular}

SP: Sepse Presente. SA: Sepse Ausente. SIDA: Síndrome da Imunodeficiência Adquirida. OR: Odds ratio. IC95\%: Intervalo de Confiança 95\%. ${ }^{*} p<0,05$ por meio do teste de qui-quadrado. Fonte: Autores (2022).

Tabela 3. Variáveis Hemodinâmicas na admissão e no desfecho.

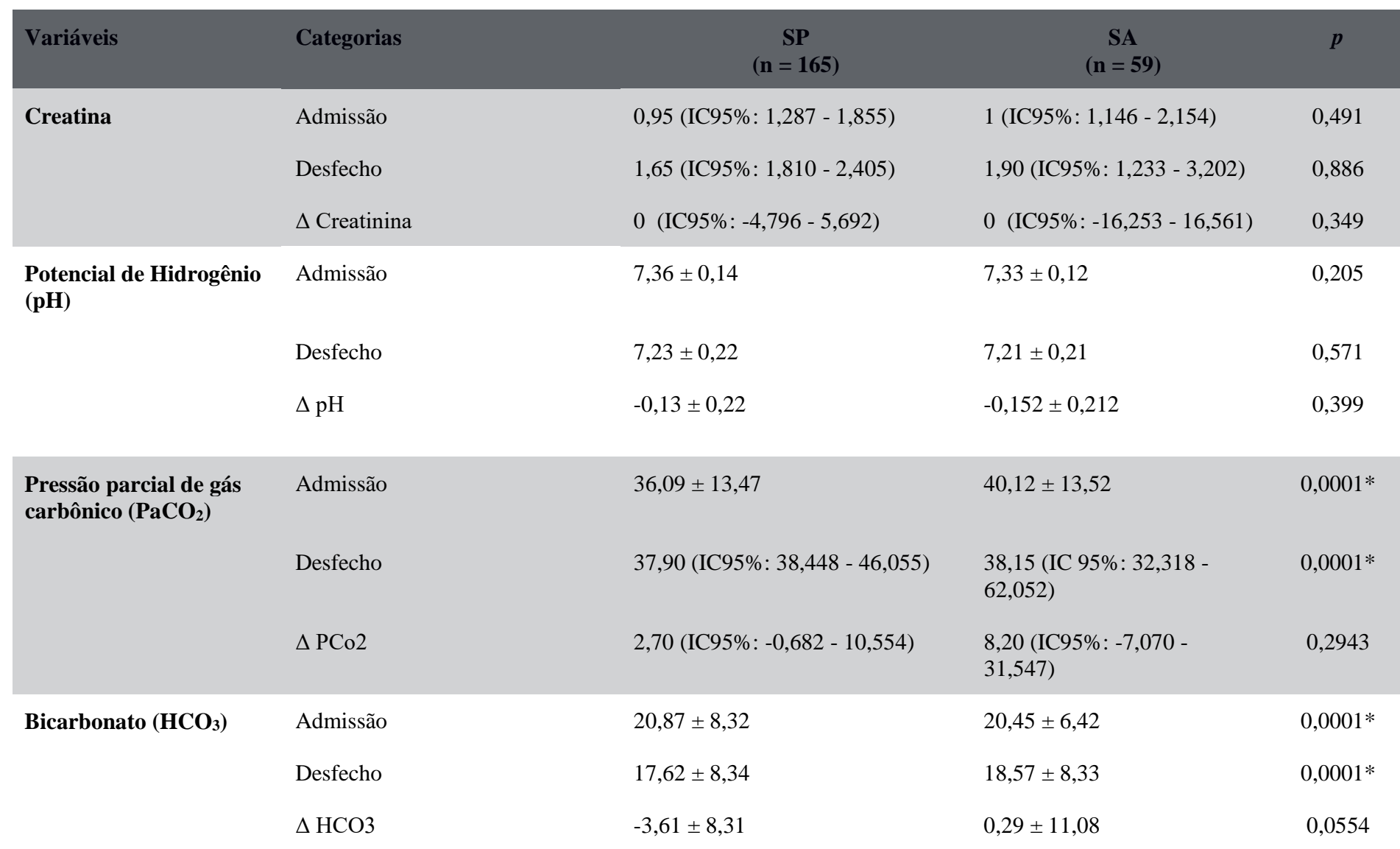




\begin{tabular}{|c|c|c|c|c|}
\hline Base (Be) & Admissão & $-3,75 \pm 9,71$ & $-2,85 \pm 7,47$ & $0,019 *$ \\
\hline \multirow[t]{2}{*}{ Leucócitos } & Admissão & 13400 (IC 95\%: 14227 - 19421) & $\begin{array}{l}15100 \text { (IC95\%: } 11480 \text { - } \\
34023)\end{array}$ & 0,1235 \\
\hline & $\Delta$ Leucócitos & 1325 (IC 95\%: 251,384 - 431,384) & $\begin{array}{l}-1285 \text { (IC } 95 \%: 154,990 \text { - } \\
334,990)\end{array}$ & 0,105 \\
\hline Plaquetas & $\Delta$ Plaquetas & $\begin{array}{l}-37500 \text { (IC 95\%: 117,351 - } \\
297,351)\end{array}$ & $\begin{array}{l}-54500(\text { IC } 95 \%: 211,935- \\
391,935)\end{array}$ & 0,346 \\
\hline \multirow[t]{3}{*}{ Hemácias } & Admissão & 3,37 (IC95\%: 3,401 - 3,733) & 3,76 (IC 95\%: 3,538 - 5,208) & $0,0001 *$ \\
\hline & Desfecho & $3,27 \pm 0,873$ & $3,97 \pm 0,976$ & $0,0001 *$ \\
\hline & $\Delta$ Hemácias & $-0,364 \pm 1,173$ & $0,123 \pm 1,191$ & 0,098 \\
\hline Ureia & Admissão & 46,50 (IC95\%: 63,507 - 87,549) & $\begin{array}{l}47,00 \text { (IC } 95 \%: 50,838- \\
90,034)\end{array}$ & $0,0001 *$ \\
\hline \multirow{2}{*}{ Sódio } & Desfecho & $144,98 \pm 10,63$ & $146,33 \pm 10,58$ & $0,0001^{*}$ \\
\hline & $\Delta$ Sódio & 3 (IC95\%: -0,402 - 10,319) & $\begin{array}{l}4,5 \text { (IC95\%: -10,293 - } \\
22,574)\end{array}$ & 0,383 \\
\hline \multirow[t]{3}{*}{ Potássio } & Admissão & 3,70 (IC95\%: 3,712 - 4,047) & 4,10 (IC95\%: 4,020 - 5,442) & $0,0006^{*}$ \\
\hline & Desfecho & 4,50 (IC95\%: 4,457 - 4,906) & 4,60 (IC95\%: 4,075 - 5,831) & 0,409 \\
\hline & $\Delta$ Potássio & 0,6 (IC95\%: -4,316 - 6,084) & $\begin{array}{l}0,5 \text { (IC95\%: -14,961 - } \\
15,448)\end{array}$ & 0,223 \\
\hline \multirow[t]{3}{*}{ Lactato } & Admissão & 1,80 (IC95\%: 2,092 - 2,660) & $1,60(\mathrm{IC} \%: 1,531-2,569)$ & 0,351 \\
\hline & Desfecho & 2,80 (IC95\%: 3,734 - 5,462) & 3,00 (IC95\%: 2,017 - 4,000) & 0,218 \\
\hline & $\Delta$ Lactato & 1 (IC95\%: -3,561 - 8,096) & $\begin{array}{l}\text { 1,30 (IC95\%: -17,446 - } \\
20,626)\end{array}$ & 0,415 \\
\hline
\end{tabular}

SP: Sepse Presente. SA: Sepse Ausente. IC95\%: Intervalo de Confiança 95\%. *p<0,05 por meio do teste de $t$ não pareado (dados paramétricos) e Mann-Whitney (dados não paramétricos). Fonte: Autores (2022). 


\section{Discussão}

A sepse é uma das principais causas de internação na UTI e da evolução ao óbito (Genga \& Russell, 2017). Foi observado no presente estudo, associação do desenvolvimento da sepse durante a internação na UTI nas seguintes variáveis sociais e clínicas.

Neste estudo, os fatores sociais escolaridade e estado civil casado foram associados à presença de sepse. De acordo com Storm et al. (2018) pacientes com escolaridade inferior a nove anos apresentam grande associação com sepse, resultado que também é observado por este estudo. Pacientes com baixa ou média escolaridade demonstram associação admissional com sepse em UTI (Storm, et al., 2018), além disso, esta população apresenta maior consumo de álcool e outras substâncias, o que está relacionado à presença de doenças crônicas e maior exposição a agentes infecciosos (Storm, et al., 2018; Oestergaard, et al., 2017; Jones, et al., 2015). Dessa forma, Oestergaard et al. (2017) aponta que existe maior risco de bacteremia por Staphylococcus aureus, um agente infeccioso que pode levar à sepse (Oestergaard, et al., 2017; Mayr et al., 2014). Com relação ao estado civil casado foi verificada a existência de associação com sepse, assim como em outro estudo prévio (Seymour, et al., 2010). Contudo, não foram encontradas na literatura justificativas para este resultado.

Quanto à mobilidade, observou-se que pacientes acamados sob cuidados intensivos estavam mais associados à sepse. Contudo, outros artigos apontam resultados diferentes, tendo em vista a busca pela restauração da homeostase fisiológica, estes pacientes são submetidos à sedação contínua e moderada, fato que se mostrou eficaz na redução da resposta ao estresse oxidativo, fornecendo conforto e facilitando o tratamento desses pacientes sépticos em estado crítico (Chen, et al., 2015; Vender, et al., 2004). No entanto, este fator não foi analisado neste estudo.

Observou-se que pacientes com sepse tiveram maior uso de ventilação mecânica. O uso de ventilação mecânica tem o intuito de reduzir o esforço e evitar fadiga da musculatura respiratória (Martin \& Grocott, 2013). Pacientes que necessitam de ventilação mecânica estão mais susceptíveis ao desenvolvimento de infecções respiratórias, pois apresentam associação à evolução com sepse (Mayr, et al., 2014). Além disso, pacientes nessa condição podem apresentar seus reflexos diminuídos como o da tosse, o que gera a diminuição do deslocamento do muco pelos cílios e consequentemente seu acúmulo nas vias aéreas, desse modo, é necessário a realização da aspiração brônquica com a finalidade de remover essas secreções (Assmann, et al., 2016). Pacientes que foram submetidos à aspiração brônquica tiveram maior incidência de sepse. Contudo, a maior frequência diária apontou uma relação com a ausência de sepse.

Neste estudo foi demonstrado que existe associação significativa entre o uso da sonda nasoentérica e a ocorrência de sepse no ambiente de terapia intensiva. A sonda nasoentérica é um tubo utilizado como suporte para a administração de nutrição enteral para pacientes que precisam de nutrição adequada diretamente ao estômago, duodeno ou jejuno, no hospital (Nguyen, 2017). No entanto, de acordo com Nguyen et al. (2017) a ocorrência de infecções em pacientes hospitalizados relacionados a nutrição está comumente relacionada a complicações provenientes do uso de outra via de administração, como a de acesso pela jugular (Nguyen, 2017).

A respeito das variáveis hemodinâmicas admissionais como $\mathrm{PaCO}_{2}, \mathrm{HCO}_{3}$ e $\mathrm{Be}$, notou-se de forma significativa distúrbios ácido-básicos, nos quais demonstra uma tendência à presença de acidose metabólica. Ademais, o estudo de Noritomi et al. (2009) mostrou concordância aos dados obtidos neste trabalho, correlacionando a admissão na UTI de pacientes sépticos com a acidose metabólica. Isso pode ser justificado pelo desequilíbrio de íons inorgânicos e associado à mortalidade, quando não foi possível eliminar a acidose (Noritomi, et al., 2009; Szrama \& Smuszkiewicz, 2016).

Os componentes do sangue como leucócitos e hemácias foram maiores no grupo SP. Na sepse o hemograma pode estar alterado, sendo um parâmetro de diagnóstico leucócitos >12000 ou >400 L (Gucyetmez \& Atalan, 2016). No estudo de Martins et al. (2019), encontrou-se que a hemoglobina foi menor nos pacientes com sepse, porém valores de leucócitos acima de 
$12.000 \mathrm{~mm} 3 / \mathrm{mL}$ foi associado como fator de risco para o desenvolvimento de sepse na UTI, corroborando com o resultado observado neste estudo.

Além disso, plaquetopenia e plaquetocitose foram observados na admissão e desfecho dos pacientes que evoluíram com sepse, respectivamente. Alguns fatores têm sido apontados na literatura como justificativa da plaquetopenia nestes pacientes, tais como: plaquetas ativadas e ligadas ao endotélio, resultando em destruição; mecanismos imunomediados; e hemofagocitose de plaquetas induzida por citocinas (Venkata, et al., 2013). Com relação à plaquetocitose no desfecho, devem ser levados em consideração os incontáveis receptores de superfície, que fazem com que as plaquetas sejam uma excepcional forma de ancoragem e transporte, que promove a disseminação de inúmeros patógenos pelo sistema corporal. Desta forma, este evento contribui para a evolução da sepse em fase final (Franchini, et al., 2017).

No presente estudo, o grupo SP apresentou redução dos níveis circulantes de sódio no desfecho. A alteração da homeostase do sódio é comum em pacientes críticos. Todavia, o aumento nos níveis de sódio tem sido apontado por outros estudos em outras circunstâncias. IJzendoorn et al. (2019) observou aumento no tecido subcutâneo. E De Freitas et al. (2019) apontou este resultado em pacientes com hipernatremia na admissão. Desta forma, não encontramos estudos que demonstrem redução dos níveis circulantes de sódio no desfecho.

Ademais, foi verificado que o aumento nos níveis de potássio e uréia alteraram de forma significativa referente aos fatores admissionais. No entanto, não encontramos pesquisas que abordassem a temática, sendo necessários mais estudos nessa área.

Além disso, pacientes em oxigenoterapia durante a admissão na UTI foi significativo para o desenvolvimento de sepse, essa terapia é comumente utilizada em pacientes internados por causa da hipoxemia, sendo necessário o suprimento de oxigênio por meio da oxigenoterapia (Martin \& Grocott, 2013). A Insuficiência Respiratória Aguda (IRA) é a principal causa que leva o paciente aos cuidados intensivos devido à necessidade de suporte ventilatório (Lai, et al., 2019). Ainda, o estado gravíssimo apresentou resultado similar, podendo ser um indicativo de uma pior evolução em curto prazo destes pacientes.

O tempo de permanência na UTI foi maior nos pacientes do grupo SP, mesmo resultado observado em outro estudo (Sakr, et al., 2018), deve-se levar em consideração que quanto maior o tempo de internação na UTI, maior a suscetibilidade de adquirir infecção hospitalar. Assim como esse estudo, na pesquisa de Mohamed et al. (2017) foi observado que pacientes com sepse tiveram maior evolução a óbito. Nesse contexto, tem sido apontado que a mortalidade está associada a fatores como baixos níveis de plaquetas, uso de ventilação mecânica invasiva (Mohamed, et al., 2017), maior tempo de internação na UTI (>28 dias), estado hemodinâmico instável (Lai, et al., 2019).

As limitações deste estudo envolveram as etapas do processo de coleta, que consiste na dificuldade ao acesso direto aos prontuários, como também a falta de informações de algumas variáveis.

\section{Considerações Finais}

Existem variáveis sociais e clínicas específicas que estão relacionadas a presença da sepse. As variáveis sociais que tiveram associação com a sepse foram a baixa escolaridade e o estado civil casado. Nas variáveis clínicas observou-se que pacientes que precisaram de intervenções invasivas fazem parte desse grupo de risco. As variáveis hemodinâmicas $\mathrm{PaCO}, \mathrm{Be}$, Bicarbonato, leucócitos, hemácias, uréia, sódio e potássio alterados foram associados ao quadro de sepse. Estes pacientes permaneceram maior tempo na UTI e tiveram maiores chances de óbito como desfecho. O conhecimento destas variáveis pode direcionar para que investimentos e recursos sejam direcionados visando prevenir esta complicação clínica.

Para perspectivas futuras, gostaríamos de atualizar o banco de dados dos pacientes para o desenvolvimento de mais publicações voltadas a essa área de atuação e assim compreender de forma mais profunda o alcance dessa temática atrelada a outras variáveis elencadas e estudadas. 


\section{Agradecimentos}

Agradecemos à Universidade Federal do Pará e ao Hospital Universitário João de Barros Barreto pelo subsídio para a pesquisa. Agradecemos também ao orientador João Simão de Melo-Neto pelo suporte e dedicação no ensino e aos demais participantes que se dedicaram na realização do trabalho. Não houve nenhum financiamento para a pesquisa.

\section{Referências}

Antal, O., Ștefănescu, E., Mleșnițe, M., Bălan, A. M., Caziuc, A. \& Hagău, N (2020). Hemodynamic Predictors for Sepsis-Induced Acute Kidney Injury: A Preliminary Study. Journal of Clinical Medicine, 9(1), 151. 10.3390/jcm9010151.

Assmann, C. B., Vieira, P. J. C., Kutchak, F., Rieder, M. M., Forgiarini, S. G. I. \& Junior, L. A. F. (2016). Lung hyperinflation by mechanical ventilation versus isolated tracheal aspiration in the bronchial hygiene of patients undergoing mechanical ventilation. Rev. bras. ter. intensiva, 28(1): 27-32. 10.5935/0103507X.20160010.

Chen, L., Meng, K., Wei, S. \& Yanjie, F. (2015). The Effect of Continuous Sedation Therapy on Immunomodulation, Plasma Levels of Antioxidants, and Indicators of Tissue Repair in Post-Burn Sepsis Patients. Cell Biochemistry and Biophysics, 73(2): 473-478. 10.1007/s12013-015-0681-x.

Da Silva, E. P., Duarte, V. C., Soares, M. M., Melo, A. T. M., Calife, E. R., Rebouças, G. S. \& De Souza, D. L. B. (2019). Survival analysis of patients with sepsis in Brazil. Rev. Soc. Bras. Med. Trop., 52: e20180121. https://doi.org/10.1590/0037-8682-0121-2018.

De Figueiredo, L. F. P., Silva, E., Correa, T. D. (2008). Avaliação hemodinâmica macro e micro-circulatória no choque séptico. Rev Med (São Paulo), abr.jun.;87(2):84-91. https://doi.org/10.11606/issn.1679-9836.v87i2p84-91

De Freitas, G., Gudu, A. \& Krishnamurthy, M. (2019). Where there is sodium there may be sepsis. Journal of Community Hospital Internal Medicine Perspectives, 9(4): 296-299. doi:10.1080/20009666.2019.1634407

Dugani, S., Veillard, J. \& Kissoon, N. (2017). Reducing the global burden of sepsis. CMAJ, 189 (1): E2-E3. 10.1503/cmaj.160798.

Engchuan, W., Dimopoulos, A. C., Tyrovolas, S., Caballero, F. F., Sanchez-Niubo, A., Arndt, H. \& Panagiotakos, D. B. (2019). Sociodemographic Indicators of Health Status Using a Machine Learning Approach and Data from the English Longitudinal Study of Aging (ELSA). Med Sci Monit., 25: 1994-2001. 10.12659/MSM.913283

Franchini, M., Veneri, D. \& Lippi, G. (2017) Thrombocytopenia and infections. Expert review of hematology, 10(1): 99-106. 10.1080/17474086.2017.1271319.

Genga, K.R. \& Russell, J.A. (2017). Update of sepsis in the intensive care unit. J Innate Immun, 2017; 9(5): 441-455. 10.1159/000477419.

Gucyetmez, B. \& Atalan, H. K. (2016). C-Reactive Protein and Hemogram Parameters for the Non-Sepsis Systemic Inflammatory Response Syndrome and Sepsis: What Do They Mean?. PLoS One, 10;11(2): e0148699. 10.1371/journal.pone.0148699.

IJzendoorn, M. V., Born, J. V. D., Hijmans, R., Bodde, R., Buter, H., Dam, W., \& Boerma, C. (2019). An observational study on intracutaneous sodium storage in intensive care patients and controls. PLoS One, 14(10): e0223100. 10.1371/journal.pone.0223100.

Jones, L., Bates, G., McCoy, E. \& Bellis, M. A. (2015). Relationship between alcohol-attributable disease and socioeconomic status, and the role of alcohol consumption in this relationship: a systematic review and meta-analysis. BMC Public Health, 15: 400. 10.1186/s12889-015-1720-7.

Lai, C.C., Tseng, K.L., Ho, C.H., Chiang, S. R, Chen, C. M, Chan, K. S. \& Cheng, K. C. (2019). Prognosis of patients with acute respiratory failure and prolonged intensive care unit stay. J Thorac Dis., 11(5):2051-2057. 10.21037/jtd.2019.04.84.

Machado, F. R., Cavalcanti, A. B., Bozza, F. A., Ferreira, E. M., Carrara, F. S. A., Sousa, J.L., \& Azevedo, L. C. P. (2017). The epidemiology of sepsis in Brazilian intensive care units (the Sepsis PREvalence Assessment Database, SPREAD): an observational study. Lancet Infect Dis, 17(11): 1180-1189. $10.1016 / \mathrm{S} 1473-3099(17) 30322-5$.

Marshall, J. C. (2018). Sepsis definitions: a work in progress. Crit Care Clin., 34(1): 1-14. 10.1016/j.ccc.2017.08.004.

Martin, D. S. \& Grocott, M. P. W. (2013). Oxygen therapy in critical illness: precise control of arterial oxygenation and permissive hypoxemia. Critical Care Med., 41(2): 423-432. 10.1097/CCM.0b013e31826a44f6.

Martins, E. C., Silveira, L. F., Viegas, K., Beck, A. D., Júnior, G. F., Cremonese, R.V., \& Lora, P. S. (2019). Neutrophil-lymphocyte ratio in the early diagnosis of sepsis in an intensive care unit: a case-control study. Rev. bras. ter. intensiva, 31(1): 64-70. 10.5935/0103-507X.20190010.

Mayr, F. B., Yende, S. \& Angus, D. C. (2014). Epidemiology of severe sepsis. Virulence, 41(2): 423-32. 10.4161/viru.27372.

Missão (2019). Hospital Universitário João de Barros Barreto - HUJBB. http://www.barrosbarreto.ufpa.br/index.php/institucional/missao.

Mohamed, A. K. S., Mehta, A. A. \& James P. (2017). Predictors of mortality of severe sepsis among adult patients in the medical Intensive Care Unit. Lung India, 34(4): 330-335. 10.4103/lungindia.lungindia_54_16.

Nguyen, D. L. (2017). Guidance for supplemental enteral nutrition across patient populations. Am J Manag Care., 23 (12 Supl): S210-S219. Retrieved from https://www.ajmc.com/view/guidance-for-supplemental-enteral-nutrition-across-patient-populations-articles 
Noritomi, D. T., Soriano, F. G., Kellum, J. A., Cappi, S. B., Biselli, P. J. C., Libório, A. B., \& Park, M. (2009). Metabolic acidosis in patients with severe sepsis and septic shock: a longitudinal quantitative study. Crit Care Med., 37(10): 2733-2739. 10.1097/ccm.0b013e3181a59165.

Oestergaard, L. B., Schmiegelow, M. D., Bruun, N. E., Skov, R. L., Petersen, A., Andersen, P. S., \& Torp-Pedersen, C. (2017). The associations between socioeconomic status and risk of Staphylococcus aureus bacteremia and subsequent endocarditis - a Danish nationwide cohort study. BMC Infectious Diseases, 17(1): 589. 10.1186/s12879-017-2691-3.

Sakr, Y., Jaschinski, U., Wittebole, X., Szakmany, T., Lipman, J., Namendys-Silva, S. A., \& Vincent, J. L. (2018). Sepsis in Intensive Care Unit Patients: Worldwide Data From the Intensive Care over Nations Audit. Open Forum Infectious Diseases, 5(12): ofy313. 10.1093/ofid/ofy313.

Salomão, R., Ferreira, B. L., Salomão, M. C., Santos, S. S., Azevedo, L. C. P. \& Brunialti, M. K. C. (2019). Sepsis: evolving concepts and challenges. Braz J Med Biol Res, 52(4): e8595. 10.1590/1414-431X20198595.

Schulz, K. F., Altman, D. G., Moher, D., for the CONSORT Group. CONSORT (2010) Statement: updated guidelines for reporting parallel group randomised trials. https://www.equator-network.org/reporting-guidelines/consort/

Seymour, C. W., Iwashyna, T. J., Cooke, C. R., Hough, C. L. \& Martin, G. S. (2010). Marital status and the epidemiology and outcomes of sepsis. Chest, 137 (6): 1289-1296. 10.1378/chest.09-2661.

Singer, M., Deutschman, C. S., Seymour, C. W., Shankar-Hari, M., Annane, D., Bauer, M., \& Angus, D. C. (2016). The third international consensus definitions for sepsis and septic shock (Sepsis-3). JAMA, 315(8): 801-810. 10.1001/jama.2016.0287.

Storm, L., Schnegelsberg, A., Mackenhauer, J., Andersen, L. W., Jessen, M. K. \& Kirkegaard, H. (2018). Socioeconomic status and risk of intensive care unit admission with sepsis. Acta Anaesthesiol Scand., 62(7): 983-992. 10.1111/aas.13114.

Szakmany, T., Lundin, R. M., Sharif, B., Ellis, G., Morgan, P., Kopczynska, M., \& Hall, J. E. (2016). Sepsis prevalence and outcome on the general wards and emergency departments in Wales: results of a multi-centre, observational, point prevalence study. PLoS One, 11(12): e0167230. 10.1371/journal.pone.0167230.

Szrama, J. \& Smuszkiewicz, P. (2016). An acid-base disorders analysis with the use of the Stewart approach in patients with sepsis treated in an intensive care unit. Anesthesiol Intensive Ther., 48(3): 180-184. 10.5603/AIT.a2016.0020.

Tsertsvadze, A., Royle, P., Seedat, F., Cooper, J., Crosby, R. \& McCarthy, N. (2016). Community-onset sepsis and its public health burden: a systematic review. Systematic Reviews, 5: 81. 10.1186/s13643-016-0243-3.

Vender, J. S., Szokol, J. W., Murphy, G. S. \& Nitsun, M. (2004). Sedation, analgesia, and neuromuscular blockade in sepsis: an evidence-based review. Critical Care Med., 32(11 Supl): S554-561. 10.1097/01.ccm.0000145907.86298.12.

Venkata, C., Kashyap, R., Farmer, J. C. \& Afessa, B. (2013). Thrombocytopenia in adult patients with sepsis: incidence, risk factors, and its association with clinical outcome. J Intensive Care., 30;1(1):9. 10.1186/2052-0492-1-9.

Westphal, G. A., Pereira, A. B., Fachin, S. M., Barreto, A. C. C., Bornschein, A, C, G, J., Filho, M. C., \& Koenig, A. (2019). Characteristics and outcomes of patients with community-acquired and hospital-acquired sepsis. Rev. bras. ter. intensiva, 31(1): 71-78. 10.5935/0103-507X.20190013. 